\title{
Broadband multilayer mirror and diffractive optics for attosecond pulse shaping in the 280-500 eV photon energy range
}

\author{
A. Guggenmos ${ }^{1,2}$, M. Hofstetter ${ }^{1,2}$, R. Rauhut ${ }^{1}$, C. Späth $^{1}$, S. Hertrich ${ }^{3}$, B. Nickel ${ }^{3}$, S. Yang ${ }^{4}$, E. M. \\ Gullikson $^{4}$, J. Schmidt ${ }^{1}$, M. Seibald ${ }^{5}$, W. Schnick ${ }^{5}$, F. Krausz ${ }^{1,2}$ and U. Kleineberg ${ }^{1,2}$ \\ ${ }^{1}$ Ludwigs-Maximilians-Universität München, Fakultät für Physik, Am Coulombwall 1, Garching, \\ Germany \\ ${ }^{2}$ Max-Planck-Institut für Quantenoptik, Hans-Kopfermann-Str. 1, Garching, Germany \\ ${ }^{3}$ Center for NanoScience (CeNS), Ludwig-Maximilians-Universität München, Schellingstraße 4, \\ München, Germany \\ ${ }^{4}$ Center for X-Ray Optics, Lawrence Berkeley National Lab 2-400, 1 Cyclotron Road, Berkeley, CA \\ 94720, USA \\ ${ }^{5}$ Ludwig-Maximilians-Universität München, Fakultät für Chemie, Butenandstr. 5-13, München, \\ Germany
}

\begin{abstract}
Chirped broadband multilayer mirrors are key components to shape attosecond pulses in the XUV range. Compressing high harmonic pulses to their Fourier limit is the major goal for attosecond physics utilizing short pulse pump-probe experiments. Here, we report about the first implementation of multilayers and diffractive optics fulfilling these requirements in the "water-window" spectral range.
\end{abstract}

\section{Introduction}

Multilayer XUV mirrors are key components for steering attosecond pulses from high harmonic radiation. These pulses pave the way towards the observation of electron dynamics in atoms, molecules or solid surfaces/nanostructures with an unprecedented temporal precision [1-3]. Extending the current technology to the "water window" photon energy range between the carbon 1s and oxygen 1s states may enable not only the generation of even shorter pulses than the current 80 as pulses achieved at $80 \mathrm{eV}$, but could also give access to deeper e-core levels and facilitate the investigation of electron dynamics in bio-molecules [4]. Aperiodic multilayer mirrors exhibit the required degree of freedom for tailored shaping of attosecond pulses in that energy range. Previous experiments have shown that multilayer mirrors can control the attosecond pulse dispersion slightly above $100 \mathrm{eV}$ [5]. Extending this control into the "water window" spectral range requires multilayer optics of "atomic" precision since the spectral phase is extremely sensitive to even smallest thickness errors.

Here we present a chirped multilayer mirror, designed via numerical algorithms, fabricated by ion beam deposition and show the excellent agreement between the simulated reflectivity, its phase and the measured XUV reflectivity [6]. Furthermore, first concepts for the design of broadband diffractive optics for nanofocusing of such attosecond XUV pulses are shown.

This is an Open Access article distributed under the terms of the Creative Commons Attribution License 2.0, which permits unrestricted use, distribution, and reproduction in any medium, provided the original work is properly cited. 


\section{Experimental methods and results}

\subsection{Deposition procedure of the multilayer}

A broadband $\mathrm{Cr} / \mathrm{Sc}$ multilayer mirror has been fabricated using a load-locked dual ion beam deposition machine, utilizing (neutralized) $600 \mathrm{eV}$ krypton ions at a background pressure of $10^{-8}$ mbar. Typical sputter rates are below $0.1 \mathrm{~nm}$ per second and have been calibrated using profilometry. The 45 degree mirror of Figure $1 \mathrm{~b}$ contains 95 layers with individual layer thicknesses ranging from $0.84 \mathrm{~nm}$ to $2.394 \mathrm{~nm}$. Each layer thickness has been controlled via deposition time based on a numerical model to compensate both for interdiffusion losses and deposition variations e.g. shutter response times.

\subsection{Comparison of XUV Measurement and Simulation}

The mirror has been designed such that its central energy coincides with the L1-edge of Argon $(326.3 \mathrm{eV})$ and to introduce an average GDD of approximately $-10000 \mathrm{as}^{2}$. A comparison of its simulated and its measured XUV (LBNL) reflectivity is shown in Figure 1a. The corresponding multilayer design is depicted in Figure 1b. An average Nevot-Croce roughness factor of $\sigma=0.573 \mathrm{~nm}$ has been included in the simulation to account for interfacial imperfections. Both the central energy and the side peaks coincide reasonably well and indicate a nearly perfect experimental implementation of the simulated multilayer stack. Assuming an attosecond pulse with a flat GDD of $+10000 \mathrm{as}^{2}$ (as from HHG plateau spectral region) being reflected off this mirror, we have derived, that it will be compressed to nearly its Fourier limit of about 65 as (FWHM) as plotted in Figure 1c. An experimental confirmation by attosecond electron streaking is planned in the near future.

a)

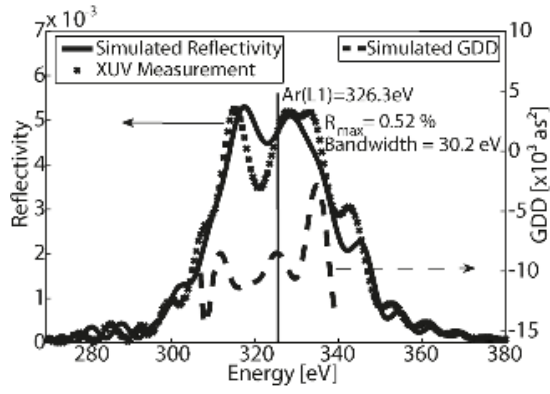

b)

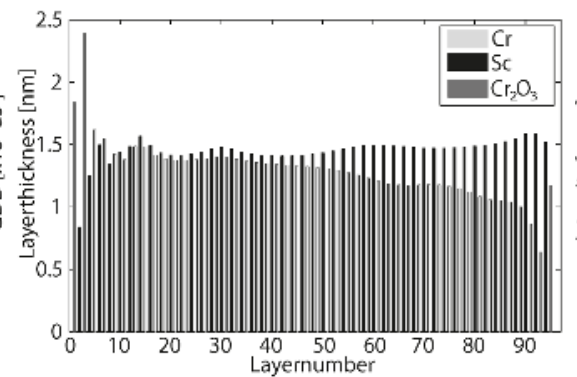

c)

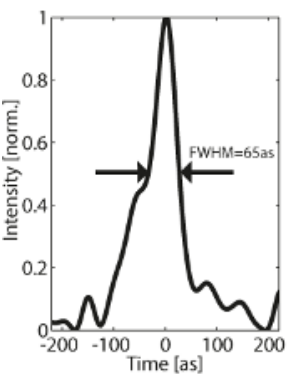

Fig. 1. a) Simulated reflectivity (black line) and measured XUV reflectivity (dotted line) with the corresponding simulated group delay dispersion in as $^{2}$ (dashed line) with a maximum reflectivity of $0.52 \%$ and a bandwidth of $30.2 \mathrm{eV}$. The black line indicates the designed central energy of $326.3 \mathrm{eV}$ and halves the reflectivity curve well. b) Multilayer design with the thicknesses of individual chromium (light grey), scandium (black) and top chromium oxide (dark grey) layer. c) Time structure of the reflected XUV pulse with a FWHM of 65 as assuming a convolution of positively chirped HHG plateau harmonics and the negatively chirped mirror.

\subsection{Diffractive optics for attosecond pulses}

Nanofocusing of attosecond soft X-ray pulses can be accomplished by means of broadband diffractive zone plate optics supporting a sufficient energy bandwidth as well as a flat spectral phase response. However, standard zone plate patterns consisting of several hundred to thousand zones do not support the required bandwidth for attosecond nanofocusing. Therefore, we designed a new type of segmented zone plate pattern consisting of four segments (Figure 1 left panel) with slightly different focused spectrum (right panel). In the focus area the spectral contributions overlap, resulting in an increased energy bandwidth of about $3.6 \mathrm{eV}$ at $326.3 \mathrm{eV}$ photon energy. The 
segmented zone plate aperture of 100 microns was designed to fit to the spatial emission of a (slightly focused) High Harmonic beam, while a diffraction limited spatial resolution of about 150 $\mathrm{nm}$ has been targeted. However, the relative placement accuracy of the outer rings of a few tens of nanometers puts stringent requirements on the e-beam lithography process.
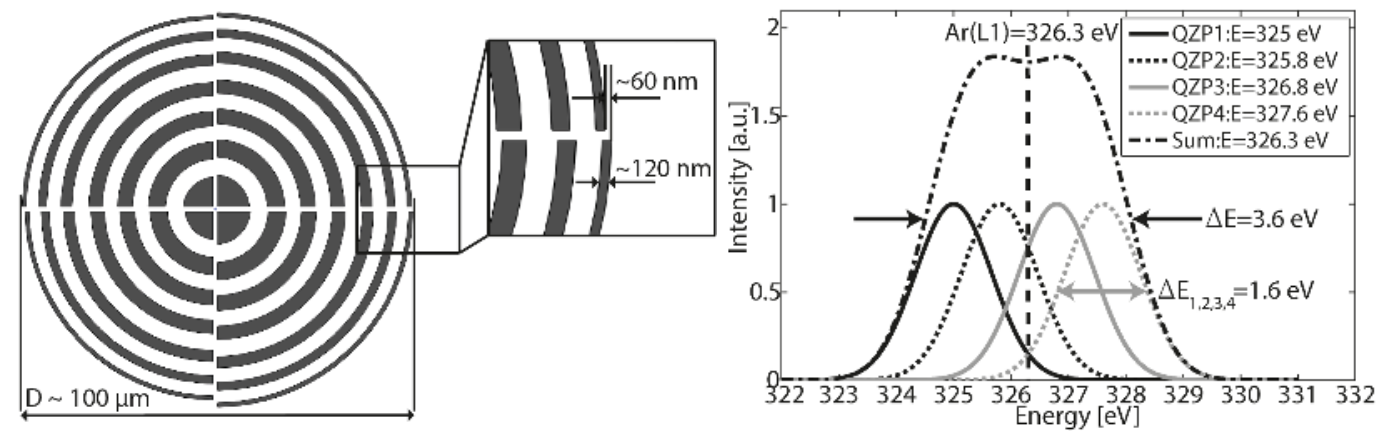

Fig. 2. Left: Quadrant zone plate (QZP) sketch with a diameter of D 100 $\mu \mathrm{m}$ and an outer zone width of $\sim 120$ $\mathrm{nm}$. The zone plates consist of 200 zones and ensure a resolution of $\sim 150 \mathrm{~nm}$. Right: Spectrum of each quadrant sector (black line, dotted black line, grey line, dotted grey line) with a bandwidth of $1.6 \mathrm{eV}$ and the convolution (dashed dotted black line) centred at the L1-Argon edge with an overall bandwidth of $3.6 \mathrm{eV}$ at $326.3 \mathrm{eV}$.

\section{Conclusions}

We have designed and realized a chirped multilayer mirror for the water window photon energy range with an averaged group delay dispersion of approximately $-10000 \mathrm{as}^{2}$ which can compress positively chirped attosecond pulses, extracted from a high harmonic plateau region, nearly to their Fourier limit of 60 as. A good agreement between the targeted and measured reflectivity was found and the desired spectral phase was derived from simulations. Direct temporal attosecond pulse measurements by attosecond electron streaking will depend on the availability of HHG sources with sufficient photon flux at a plateau area reaching up to $\sim 350 \mathrm{eV}$ and are expected for the near future. In combination with the introduced segmented zone plate these two independent attosecond optics may serve as tools for steering an attosecond pulse with novel spectral and spatial accuracy.

\section{References}

1. M. Uiberacker, T. Uphues, M. Schultze, A. J. Verhoef, V. Yakovlev, M. F. Kling, J. Rauschenberger, N. M. Kabachnik, H. Schröder, M. Lezius, K. L. Kompa, H.-G. Muller, M. J. J. Vrakking, S. Hendel, U. Kleineberg, U. Heinzmann, M. Drescher, F. Krausz, Nature, 446, 627632 (2007)

2. M. Drescher, M. Hentschel, R. Kienberger, M. Uiberacker, V. Yakovlev, A. Scrinzi, T. Westerwalbesloh, U. Kleineberg, U. Heinzmann, F. Krausz, Nature, 419, 803-807 (2002)

3. M. Schultze, M. Fieß, N. Karpowicz, J. Gagnon, M. Korbman, M. Hofstetter, S. Neppl, A. L. Cavalieri, Y. Komninos, T. Mercouris, C. A. Nicolaides, R. Pazourek, S. Nagele, J. Feist, J. Burgdörfer, A. M. Azzeer, R. Ernstorfer, R. Kienberger, U. Kleineberg, E. Goulielmakis, F. Krausz, V. S. Yakovlev, Science, 328, 1658-1662 (2010)

4. E. Goulielmakis, M. Schultze, M. Hofstetter, V. S. Yakovlev, J. Gagnon, M. Uiberacker, A. L. Aquila, E. M. Gullikson, D. T. Attwood, R. Kienberger, F. Krausz, U. Kleineberg, Science, 320, 1614-1617 (2008)

5. M. Hofstetter, M. Schultze, M. Fieß, B. Dennhardt, A. Guggenmos, J. Gagnon, V. S. Yakovlev, E. Goulielmakis, R. Kienberger, E. M. Gullikson, F. Krausz, U. Kleineberg, Optics Express, 19, 1767-1776 (2011)

6. A. V. Tikhonravov, M. K. Trubetskov, G. W. DeBell, Appl. Opt., 46, 704-710 (2007) 